\title{
ВЛИЯНИЕ СПОСОБА АОРТОКОРОНАРНОГО ШУНТИРОВАНИЯ ПРИ МНОГОСОСУДИСТОМ ПОРАЖЕНИИ КОРОНАРНЫХ АРТЕРИЙ НА ПОСЛЕДУЮЩЕЕ КАЧЕСТВО ЖИЗНИ БОЛЬНЫХ
}

\author{
( Суковатых Б.С., Назаренко П.М., Григорьев Н.Н., Суковатьх М.Б., \\ Горпинич А.Б., Полянский Д.В., Мишустин В.Н.
}

\author{
Курский государственный медицинский университет (КГМУ) \\ Россия, 305041, Курская область, г. Курск, ул. К. Маркса, д. 3
}

\begin{abstract}
Цель работы: сравнить динамику показателей качества жизни больных ишемической болезнью сердца (ИБС) после аортокоронарного шунтировани (АКШ) на работающем сердце и с применением искусственного кровообращения (ИК) и на этой основе определить наиболее оптимальный выбор операции в зависимости от прогнозируемой длительности жизни больного.
\end{abstract}

Материалы и методы. Изучено качество жизни 40 пациентов после аортокоронарного шунтирования. Пациенты были разделены на 2 группы по 20 человек в каждой. В первой группе операции проводились на работающем сердце, а во второй - с применением искусственного кровообращения. Качество жизни больных до и после операции через 6 и 12 месяцев изучено с помощью опросника SF-36.

Результаты. У больных перед операцией физический компонент здоровья снижен на 23,6士3,3, а психологческий - на 10,2 $\pm 3,1$ по сравнению со здоровой популяцией жителей России (p<0,05). Через 6 месяцев после операции

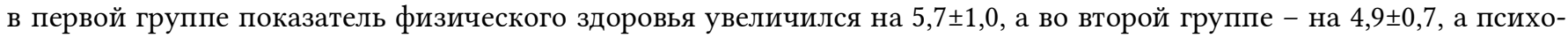

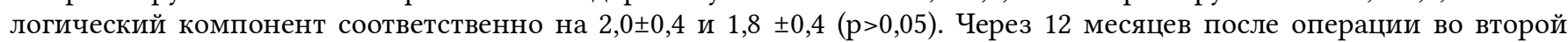
группе показатели физического компонента здоровья превышали соответствующий показатель в первой группе

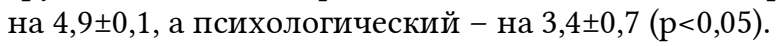

Заключение. Операции на работающем сердце возможно проводить у пациентов с короткой прогнозируемой продолжительностью жизни при фракции выброса левого желудочка менее $40 \%$.

Ключевые слова: ишемическая болезнь сердца; многососудистое поражение коронарных артерий; аортокоронарное шунтирование; работающее сердце; искусственное кровообращение; качество жизни.

Суковатых Борис Семенович - д-р мед. наук, профессор, зав. кафедрой общей хирургии, КГMУ, г. Kypcк. ORCID iD: 0000-0003-2197-8756. E-mail: sukovatykhbs@kursksmu.net (автор, ответственный за переписку)

Назаренко Петр Михайлович - д-р мед. наук, профессор, зав. кафедрой хирургических болезней № 2, КГМУ, г. Курск. ORCID iD: 0000-0003-4961-1382. E-mail: nazarenkopm@kursksmu.net

Григорьев Николай Николаевич - д-р мед. наук, профессор кафедры хирургических болезней ФПО, КГМУ, г. Курск. ORCID iD: 0000-0002-4102-1516. E-mail: grigorevnn@kursksmu.net

Суковатых Михаил Борисович - канд. мед. наук, доцент кафедры общей хирургии, КГМУ, г. Курск. ORCID iD: 0000-00031907-4395. E-mail: sukovatykhmb@kursksmu.net

Горпинич Александр Борисович - д-р мед. наук, профессор, профессор кафедры общей хирургии, КГМУ, г. Курск. E-mail: doc.goralex@mail.ru

Полянский Дмитрий Владимирович - ординатор кафедры общей хирургии, КГМУ, г. Курск. ORCID iD: 0000-0003-30778801. E-mail: polyanskydmitriy@yandex.ru

Мишустин Владимир Николаевич - д-р мед. наук, профессор, профессор кафедры хирургических болезней ФПО, КГМУ, г. Курск. ORCID iD: 0000-0002-4104-5681. E-mail: vladimirshef2011@yandex.ru

Нет ни одной страны в мире, в которой болезни системы кровообращения не стояли бы на первом месте по причинам смертности населения. В России от этих заболеваний ежегодно умирают около 860 тыс. человек [3]. На первом месте среди сердечно-сосудистой патологии по заболеваемости, срокам утраты трудоспособности и смертности, как в России, так и в США, находится ишемическая болезнь сердца (ИБС) [2]. На начало 2019 г. базовое значение показателей смертности от сердечно-сосудистых заболеваний составляет 587,6 на 100 тыс населения России. Национальный проект «Здравоохранение» предусматривает меры по снижению этого показателя к 2024 г. до 450,0, т.е. в 1,3 раза.
Для реваскуляризации миокарда применяют медикаментозное лечение, чрескожные коронарные вмешательства (ЧКВ) и аортокоронарное шунтирование (АКШ). До настоящего времени при многих клинических ситуациях нет четких доказательств преимущества того или иного способа реваскуляризации миокарда [1]. Нерешенной проблемой является лечение пациентов с множественными атеросклеротическими поражениями коронарных артерий $[7,8]$. Существуют два подхода к хирургическому лечению: АКШ с применением искусственного кровообращения (ИК) и АКШ на работающем сердце. Предметом дискуссии является выбор методики хирургической реваскуляризации миокарда. АКШ с применением ИК 
бесспорно является более траматической операцией, чем операция на работающем сердце. АКШ на работающем сердце хотя и позволяет избежать негативного влияния ИК, но в техническом плане является более сложной, но менее травматичной операцией. Летальность после этих операций в ближайшем послеоперационном периоде оказалась одинаковой и колебалась от $3 \%$ до $3,5 \%[6,9,10]$.

При изучении выживаемости больных в отдаленном периоде после АКШ на работающем сердце и после традиционного АКШ также не установлено существенных различий. Однако было подчеркнуто, что при выборе способа операции необходимо учитывать тяжесть сопутствующей соматической патологии и диффузность поражения коронарного русла [5, 11, 12].

Качество жизни (КЖ) пациентов является одним из основных показателей, характеризующих эффективность проведенного лечения. В России многие хирурги скептически относятся к оценке результатов лечения по качеству жизни больных, считая его субъективным показателем, зависящим от оценки самого больного влияния операции на его последующую жизнь. Предпочтение отдается объективным результатам инструментального обследования сердечнососудистой системы пациента. Вместе с тем во всех сферах жизнедеятельности человека качество оказанной ему услуги определяет сам человек, получивший ее, а не тот, который ее предоставил. В литературе отсутствуют сведения о сравнительном изучении качества жизни больных, перенесших операции с применением ИК и на работающем сердце. Определение различий в качестве жизни больных, в зависимости от способа АКШ, позволит целенаправленно определять выбор операции исходя из прогнозируемой продолжительности жизни больного. Чем короче продолжительность, тем менее травматичной должна быть операция. По данным Фремингемского исследования, одним из основных факторов определения продолжительности жизни больного с ИБС является фракция выброса левого желудочка, критический показатель которого - 40\%. [4] В России представлено несколько десятков опросников качества жизни больных, адаптированных к определенным соматическим заболеваниям. С нашей точки зрения, для этих целей лучше использовать опросник, который нашел наибольшее распространение в мировой практике.

Цель исследования: сравнить динамику показателей качества жизни больных ИБС после АКШ на работающем сердце и с применением ИК и на этой основе определить наиболее опти- мальный выбор операции в зависимости от прогнозируемой длительности жизни больного.

\section{МАТЕРИАЛЫ И МЕТОДЫ ИССЛЕДОВАНИЯ}

Проведено изучение качества жизни 40 пациентов, поровну мужчин и женщин, среднего и пожилого возраста, страдающих ИБС, которым было проведено АКШ в ФГБУ «Национальный медико-хирургический Центр имени Н.И. Пирогова». Критерии включения: информированное согласие пациента; стабильная и нестабильная стенокардия, рефрактерная к медикаментозной терапии и ухудшающая качество жизни больных; множественное атеросклеротическое поражение проксимальных отделов коронарных артерий с суммарным стенозом $70 \%$ и более просвета; острая ишемия или нестабильность гемодинамики после неудачной попытки ангиопластики или стентирования с развивающимся инфарктом миокарда в течение 4-6 часов от начала грудной боли. Критерии исключения: острый период нарушения мозгового кровообращения; анемии различного генеза; злокачественная неконтролируемая артериальная гипертензия; инфекционные заболевания; тяжелые соматические заболевания в терминальных стадиях.

Выделены две равных группы по 20 человек в каждой, сопоставимые по половозрастному составу, степени поражения коронарных артерий, функциональному состоянию миокарда. Больным в первой группе выполнено АКШ на работающем сердце, а во второй - АКШ с применением ИК. В качестве контроля использовали показатели КЖ 20 здоровых лиц, не страдающих ИБС. Изучение параметров качества жизни больных проводилось через 6 и 12 месяцев после операции с помощью опросника SF-36. Опросник состоит из восьми шкал. Первые четыре шкалы отражают физический статус больного, а вторые черые - психологический статус. На основании этих шкал формируются два объединенных показателя: физический и психологический компоненты здоровья. Этот опросник наиболее часто применяется во всем мире для оценки КЖ.

Количественные результаты исследования обрабатывали статистически с использованием аналитического пакета приложения ExcelOffice 2010. Проверку на нормальность распределения проводили по способу Шапиро-Уилка. При нормальном распределении статистическую значимость различий осуществляли с помощью критерия Стьюдента. Полученные данные представлены в виде $\mathrm{M} \pm \mathrm{m}$. Различия считали статистически достоверными при $\mathrm{p}<0.05$. 


\section{РЕЗУЛЬТАТЫ ИССЛЕДОВАНИЯ И ИХ ОБСУЖДЕНИЕ}

Показатели качества жизни больных контрольной и исследуемых групп до операции представлены в таблице 1.

При сравнении показателей КЖ больных, страдающих ИБС, с показателями людей того же возраста и пола, но не имеющих проблем с состоянием сердечно-сосудистой системы, установлены достоверные различия по всем показателям. ИБС негативно влияет как на физический, так и на психологический статус человека. Больные снижают в 1,5 раза интенсивность профессиональной и непрофессиональной деятельности, болевые ощущения возрастают при нагрузке в 1,6 раза, а общее состояние здоровья уменьшается в 1,4 раза. Жизненная активность падает в 1,15 раза, социальное функционирование - в 1,14 раза, эмоциональное функционирование - в 1,2 раза, психическое здоровье - в 1,1 раза. Физический компонент здоровья перед операцией снижается на $23,6 \pm 3,3$, а психологи-

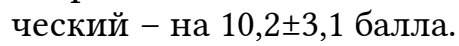

Динамика показателей качества жизни через 6 месяцев после операции представлена в таблице 2.
Через 6 месяцев после операции у больных в первой группе динамика показателей качества жизни была следующей: первый показатель возрос на $5,2 \%$, второй и третий - на $5,4 \%$, четвертый - на $1,7 \%$, пятый - на $5,8 \%$, шестой - на $0,5 \%$, седьмой - на $0,8 \%$, восьмой - на $1,4 \%$, девятый - на $5,7 \%$, а десятый - на $2,0 \%$. Во второй группе показатели возросли следующим образом: первый показатель - на 4,9\%, второй - на $5,1 \%$, третий - на $4,7 \%$, четвертый - на $2,9 \%$, пятый - на $6,1 \%$, шестой - на $1,1 \%$, седьмой - на $0,6 \%$, восьмой - на $0,8 \%$, девятый - на $4,9 \%$, а десятый - на $1,8 \%$.

Достоверные различия по сравнению с дооперационными выявлены как в первой, так и во второй группе по следующим шкалам: физическое функционирование, ролевой фактор, фактор боли и жизненная активность. Из них первые три фактора характеризуют физический компонент здоровья, который оказался достоверно выше по сравнению с дооперационным в обеих группах больных (p<0.05). Факторы качества жизни, характеризующие психологическое здоровье, за исключением жизненной активности, достоверно не изменились ( $>>0.05)$.

Таблица 1 Table 1

Качество жизни больных до оперативного лечения

Patients' quality of life before surgical treatment

\begin{tabular}{|c|c|c|c|c|}
\hline № & $\begin{array}{l}\text { Показатели } \\
\text { Items }\end{array}$ & $\begin{array}{c}\text { Контрольная группа } \\
\text { Control group } \\
(\mathrm{n}=20)\end{array}$ & $\begin{array}{l}1 \text { группа } \\
1^{\text {st }} \text { group } \\
(\mathrm{n}=20)\end{array}$ & $\begin{array}{l}2 \text { группа } \\
2^{\text {nd }} \text { group } \\
(\mathrm{n}=20)\end{array}$ \\
\hline 1 & $\begin{array}{l}\text { Физическое функционирование } \\
\text { Physical functioning }\end{array}$ & $69.2 \pm 7.4$ & $44.6 \pm 1.9^{*}$ & $45.2 \pm 3.2^{*}$ \\
\hline 2 & $\begin{array}{l}\text { Ролевое физическое функционирование } \\
\text { Role physical functioning }\end{array}$ & $64.4 \pm 8.2$ & $43.4 \pm 2.6^{*}$ & $42.8 \pm 3.1^{*}$ \\
\hline 3 & $\begin{array}{l}\text { Физическая боль } \\
\text { Bodily pain }\end{array}$ & $70.8 \pm 5.6$ & $44.1 \pm 2.2^{*}$ & $43.9 \pm 1.6^{*}$ \\
\hline 4 & $\begin{array}{l}\text { Общее состояния здоровья } \\
\text { General health perceptions }\end{array}$ & $66.2 \pm 9.4$ & $48.5 \pm 4.6^{*}$ & $46.9 \pm 4.2^{*}$ \\
\hline 5 & $\begin{array}{l}\text { Жизненная активность } \\
\text { Vitality }\end{array}$ & $58.4 \pm 4.8$ & $42.6 \pm 1.8^{*}$ & $43.1 \pm 0.7^{*}$ \\
\hline 6 & $\begin{array}{l}\text { Социальное функционирование } \\
\text { Social functioning }\end{array}$ & $61.8 \pm 6.2$ & $53.3 \pm 2.6^{*}$ & $54.1 \pm 4.3^{*}$ \\
\hline 7 & $\begin{array}{l}\text { Ролевое эмоциональное функционирование } \\
\text { Role emotional functioning }\end{array}$ & $62.1 \pm 4.9$ & $51.2 \pm 5.4^{*}$ & $52.1 \pm 3.2^{*}$ \\
\hline 8 & $\begin{array}{l}\text { Психическое здоровье } \\
\text { Mental health }\end{array}$ & $60.6 \pm 7.8$ & $53.4 \pm 2.1^{*}$ & $52.8 \pm 3.0^{*}$ \\
\hline 9 & $\begin{array}{l}\text { Физический компонент здоровья } \\
\text { Physical health self-assessment }\end{array}$ & $67.6 \pm 6.1$ & $43.8 \pm 3.2^{*}$ & $44.2 \pm 2.4^{*}$ \\
\hline 10 & $\begin{array}{l}\text { Психологический компонент здоровья } \\
\text { Mental health self-assessment }\end{array}$ & $60.7 \pm 5.4$ & $50.2 \pm 2.3^{*}$ & $50.8 \pm 1.9^{*}$ \\
\hline
\end{tabular}

Примечание: здесь и в таблице 3: * - статистически значимая разница в сравнении с контрольной группой $(\mathrm{p} \leq 0,05)$.

Note: here and in table $3:{ }^{*}$ - statisticaly accurate difference compared with control group $(\mathrm{p} \leq 0.05)$. 
Динамика качества жизни через 6 месяцев после операции

Patients' quality of life 6 months after surgery

\begin{tabular}{|c|c|c|c|}
\hline № & $\begin{array}{l}\text { Показатели } \\
\text { Items }\end{array}$ & $\begin{array}{c}\text { группа } \\
1^{\text {st }} \text { group } \\
(\mathrm{n}=20)\end{array}$ & $\begin{array}{c}2 \text { группа } \\
2^{\text {st }} \text { group } \\
(\mathrm{n}=20)\end{array}$ \\
\hline 1 & $\begin{array}{l}\text { Физическое функционирование } \\
\text { Physical functioning }\end{array}$ & $49.8 \pm 2.8$ & $50.1 \pm 1.2$ \\
\hline 2 & $\begin{array}{l}\text { Ролевое физическое функционирование } \\
\text { Role physical functioning }\end{array}$ & $48.8 \pm 2.9$ & $47.9 \pm 2.7$ \\
\hline 3 & $\begin{array}{l}\text { Физическая боль } \\
\text { Bodily pain }\end{array}$ & $49.5 \pm 2.6$ & $48.6 \pm 1.4$ \\
\hline 4 & $\begin{array}{l}\text { Общее состояния здоровья } \\
\text { General health perceptions }\end{array}$ & $50.2 \pm 2.1$ & $49.8 \pm 2.8$ \\
\hline 5 & $\begin{array}{l}\text { Жизненная активность } \\
\text { Vitality }\end{array}$ & $48.4 \pm 1.9$ & $49.2 \pm 1.1$ \\
\hline 6 & $\begin{array}{l}\text { Социальное функционирование } \\
\text { Social functioning }\end{array}$ & $53.8 \pm 1.6$ & $55.2 \pm 3.8$ \\
\hline 7 & $\begin{array}{l}\text { Ролевое эмоциональное функционирование } \\
\text { Role emotional functioning }\end{array}$ & $52.6 \pm 2.4$ & $52.9 \pm 2.7$ \\
\hline 8 & $\begin{array}{l}\text { Психическое здоровье } \\
\text { Mental health }\end{array}$ & $54.2 \pm 1.6$ & $53.4 \pm 1.8$ \\
\hline 9 & $\begin{array}{l}\text { Физический компонент здоровья } \\
\text { Physical health self-assessment }\end{array}$ & $49.5 \pm 2.2$ & $49.1 \pm 1.7$ \\
\hline 10 & $\begin{array}{l}\text { Психологический компонент здоровья } \\
\text { Mental health self-assessment }\end{array}$ & $52.2 \pm 1.9$ & $52.6 \pm 2.3$ \\
\hline
\end{tabular}

Динамика качества жизни через 12 месяцев после операции

Patients' quality of life 12 months after surgery

\begin{tabular}{|c|c|c|c|}
\hline № & $\begin{array}{l}\text { Показатели } \\
\text { Items }\end{array}$ & $\begin{array}{c}\text { группа } \\
1^{\text {st }} \text { group } \\
(\mathrm{n}=20)\end{array}$ & $\begin{array}{c}2 \text { группа } \\
2^{\text {st }} \text { group } \\
(n=20)\end{array}$ \\
\hline 1 & $\begin{array}{l}\text { Физическое функционирование } \\
\text { Physical functioning }\end{array}$ & $62.2 \pm 2.8$ & $67.7 \pm 3.1^{*}$ \\
\hline 2 & $\begin{array}{l}\text { Ролевое физическое функционирование } \\
\text { Role physical functioning }\end{array}$ & $58.6 \pm 2.1$ & $62.4 \pm 1.8^{*}$ \\
\hline 3 & $\begin{array}{l}\text { Физическая боль } \\
\text { Bodily pain }\end{array}$ & $60.4 \pm 1.9$ & $66.1 \pm 2.4^{*}$ \\
\hline 4 & $\begin{array}{l}\text { Общее состояния здоровья } \\
\text { General health perceptions }\end{array}$ & $59.3 \pm 2.8$ & $61.9 \pm 2.6$ \\
\hline 5 & $\begin{array}{l}\text { Жизненная активность } \\
\text { Vitality }\end{array}$ & $53.1 \pm 4.2$ & $56.3 \pm 1.9^{*}$ \\
\hline 6 & $\begin{array}{l}\text { Социальное функционирование } \\
\text { Social functioning }\end{array}$ & $58.4 \pm 2.3$ & $60.8 \pm 1.2$ \\
\hline 7 & $\begin{array}{l}\text { Ролевое эмоциональное функционирование } \\
\text { Role emotional functioning }\end{array}$ & $59.6 \pm 1.2$ & $61.1 \pm 0.9$ \\
\hline 8 & $\begin{array}{l}\text { Психическое здоровье } \\
\text { Mental health }\end{array}$ & $57.4 \pm 2.3$ & $60.1 \pm 0.4^{*}$ \\
\hline 9 & $\begin{array}{l}\text { Физический компонент здоровья } \\
\text { Physical health self-assessment }\end{array}$ & $59.6 \pm 2.4$ & $64.5 \pm 2.5^{*}$ \\
\hline 10 & $\begin{array}{l}\text { Психологический компонент здоровья } \\
\text { Mental health self-assessment }\end{array}$ & $56.1 \pm 1.9$ & $59.5 \pm 1.2^{*}$ \\
\hline
\end{tabular}


При сравнении показателей качества жизни между группами больных достоверных различий не выявлено.

Следовательно, технология проведения АКШ через 6 месяцев после операции существенно не влияет на качество жизни больных. Следует подчеркнуть, что травматичность оперативного лечения значительно удлиняет период реабилитации больных и показатели качества жизни через 6 месяцев после операции остаются достаточно низкими.

Динамика показателей качества жизни через 12 месяцев после операции представлена в таблице 3.

Через 12 месяцев по сравнению с 6-месячным сроком отмечено улучшение качества жизни больных в обеих группах. Так, в первой группе первый показатель увеличился на 16,4\%, а во второй группе - на 17,6\%, второй показатель соответственно на $9,8 \%$ и $14,5 \%$, третий - на $10,9 \%$ и $17,5 \%$, четвертый - на $9,1 \%$ и $12,1 \%$, пятый - на $4,7 \%$ и $7,1 \%$, шестой - на $4,6 \%$ и $5,6 \%$, седьмой на $7,0 \%$ и $8,2 \%$, восьмой - на $3,2 \%$ и $6,7 \%$, девятый - на $10,1 \%$ и 15,4 , десятый - на $3,9 \%$ и $6,9 \%$.

Выявлены более высокие показатели КЖ во второй группе по сравнению с первой: физический фактор - на 5,5\%; ролевой фактор - на $3,8 \%$; фактор боли - на 5,7\%; жизненная активность на $3,2 \%$, физический компонент здоровья $-4,9 \%$, психологический компонент здоровья - 3,4\% $(\mathrm{p}<0,05)$. Статистически достоверных различий между остальными показателями не выявлено.

Полученные результаты свидетельствуют, что через год после выполнения операции выбор способа влияет на последующее качество жизни больных.

На этом сроке показатели качества жизни больных после АКШ с применением ИК достоверно выше, чем после АКШ на работающем сердце, что обусловлено более полной реваскуляризацией миокарда. При многососудистом поражении коронарных артерий АКШ, выполненное с применением ИК, позволяет добиться полной реваскуляризации миокарда. Поэтому при отсутствии противопоказаний к применению ИК у больных с низким операционным риском при фракции выброса левого желудочка более $40 \%$ следует отдавать предпочтение традиционному аортокоронарному шунтированию. У больных в группе высокого риска выполнение АКШ на работающем сердце позволяет при многососудистом поражении коронарных артерий лишь улучшить кровоснабжение отдельных отделов миокарда, что мало влияет на показатели качества жизни. Поэтому АКШ на работающем сердце следует выполнять у больных с короткой прогнозируемой жизнью, при выраженном поражении восходящей аорты, дисфункции левого желудочка (фракция выброса менее 40\%), почечной, печеночной, дыхательной, церебральной недостаточностью.

Таким образом, качество жизни больных через 1 год после операции на работающем сердце при многососудистых поражениях достоверно ниже, чем после АКШ с применением ИК. Применять традиционное АКШ возможно у пациентов с низким операционным риском, а АКШ на работающем сердце выполнять у больных в группе высокого операционного риска с прогнозируемой короткой продолжительностью жизни.

\section{КОНФЛИКТ ИНТЕРЕСОВ}

Авторы заявляют об отсутствии конфликта интересов.

\section{ИСТОЧНИКИ ФИНАНСИРОВАНИЯ}

Авторы заявляют об отсутствии финансирования.

\section{СООТВЕТСТВИЕ ПРИНЦИПАМ ЭТИКИ}

Авторы подтверждают, что в соответствии с Хельсинкской декларацией соблюдены права людей, принимавших участие в исследовании, включая получение информированного согласия. Дизайн исследования одобрен Региональным этическим комитетом при Курском государственном медицинском университете (Протокол № 5 от 11.05.2015).

\section{ЛИЧНЫЙ ВКЛАД АВТОРОВ}

Суковатых Б.С. - разработка концепции и дизайна; Назаренко П.М. - анализ литературных данных по теме исследования; Григорьев Н.Н. - анализ полученных результатов исследования; Суковатых М.Б. - написание статьи; Горпинич А.Б. - анализ полученных результатов исследования; Полянский Д.В. - опрос больных по шкале SF-36; Мишустин В.Н. - анализ литературных данных по теме исследования.

\section{ЛИТЕРАTУРA / REFERENSES}

1. Акчурин Р.С., Ширлев А.А., Васильев В.П., Галятдинов Д.М., Власов Э.Е. Современные тенденции в коронарной хирургии. Патология кровообращения и кардиохирургия. 2017; 21(3S):34-44 [Akchurin Renat S., Shiryaev A.A., Vasiliev Vladislav P., Galyautdinov D.M., Vlasova E.E. Modern trends in coronary surgery. Patologiya krovoobrashcheniya i kardiohirurgiya. 2017; 21(3S):34-44 (in Russ.)] DOI: $10.21688 / 1681-3472-2017-3 S-34-44$

2. Бойцов С.А., Зайратьянс О.В., Андреев Е.Н., Самородская И.В. Сравнение показателей смертности от ишемической болезни сердца среди мужчин и женщин старше 50 лет в России и США. Российский кардиологический журнал. 2017; 22(6):100-107 [Boytsov S.A., Zayratiants O.V., Andreev E.M., Samorodskaya I.V. Comparison of coromary heart disease mortality in men and women age 
50 years and older in Russia and USA. Russian journal of cardiology. 2017; 22(6): 100-107 (in Russ.)] DOI: 10.15829/1560-4071-2017-6-100-107

3. Чазова И.Е., Ощепкова И.В. Борьба с сердечнососудистыми заболеваниями: проблемы и пути их решения на современном этапе. Вестник Росздравнадзора. 2015; 5:7-10 [Chazova I.E., Oschepkova E.V. The fight against cardiovascular diseases: problems and solutions at the present stage. Vestnik Roszdravnadzora. 2015; 5:7-10. (in Russ.)]

4. Andersson C., Lin H., Liu C., Levy D., Mitchell G.F., Larson M.G., Vasan R.S. An Integrated Multi-Omics Approach to Identify Genetic Underpinnings of Heart Failure and its Echocardiographic Precursors: The Framingham Heart Study. Circ Genom Precis Med. 2019. DOI: $10.1161 /$ CIRCGEN.118.002489

5. Calafiore A.M., Prapas S., Osman A., Di Mauro M. Coronary artery bypass grafting off-pump or onpump: another brick in the wall. Ann Transl Med. 2017; 5(7):168. DOI: 10.21037/atm.2017.03.52

6. Chaudhry U.A., Harling L., Sepehripour A.H., Stavridis G., Kokotsakis J., Ashrafian H., Athanasiou T. Beating-Heart Versus Conventional On-Pump Coronary Artery Bypass Grafting: A Meta-Analysis of Clinical Outcomes. Ann Thorac. Surg. 2015; 100(6):2251-2260. DOI: 10.1016/j.athoracsur.2015.05.057

7. Chikwe J., Sun E., Hannan E.L., Itagaki S., Lee T., Adams D.H., Egorova N.N. Outcomes of Second Arterial Conduits in Patients Undergoing Multivessel Coronary Artery Bypass Graft Surgery. $7 \mathrm{Am}$ Coll
Cardiol. 2019; 74(18):2238-2248. DOI: 10.1016/j.jacc.2019.08.1043

8. Hayatsu Y., Ruel M., Sun L.Y. Renal insufficiency and severe coronary artery disease: should coronary artery bypass grafting, off-pump coronary artery bypass grafting or percutaneous coronary intervention be performed? Curr Opin Cardiol. 2019; 34(6):645-649. DOI: 10.1097/HCO.0000000000000687

9. Kirmani B.H., Holmes M.V., Muir A.D. Long-term survival and freedom from reintervention after offpump coronary artery bypass grafting: a propensitymatched study. Circulation. 2016; 134(17):1209-1220. DOI: 10.1161/CIRCULATIONAHA.116.021933

10. Lamy A., Devereaux P.J., Prabhakaran D., Taggart D.P., Hu S., Straka Z., Piegas L.S., Avezum A. et al. Five-year outcomes after offpump or on-pump coronary-artery bypass grafting. N Engl f Med. 2016; 375(24):2359-2368. DOI: 10.1056/NEJMoa1601564

11. Reents W., Zacher M., Boergermann J., Kappert U., Hilker M, Färber G., Albert M., Holzhey D. et al. OffPump Coronary Artery Bypass Grafting and StrokeExploratory Analysis of the GOPCABE Trial and Methodological Considerations. Thorac Cardiovasc Surg. 2018; 66(6):464-469. DOI: 10.1055/s-00381636936

12. Tachibana H., Hiraoka A., Saito K., Naito Y., Chikazawa G., Tamura K., Totsugawa T., Yoshitaka $\mathrm{H}$. et al. Incidence and impact of silent brain lesions after coronary artery bypass grafting. 7 Thorac Cardiovasc Surg. 2019; 158(4):978-984. DOI: 10.1016/j.jtcvs.2019.09.162

Для цитирования: Суковатых Б.С., Назаренко П.М., Григорьев Н.Н., Суковатых М.Б., Горпинич А.Б., Полянский Д.В., Мишустин В.Н. Влияние способа аортокоронарного шунтирования при многососудистом поражении коронарных артерий на последующее качество жизни больных. Курский научно-практический вестник «Человек и его здоровье». 2019 ; (3):56-62. DOI: $10.21626 /$ vestnik/2019-3/08. 


\title{
OPTIONS OF THE CORONARY ARTERY BYPASS GRAFTING TYPE IN PATIENTS WITH MULTIVASCULAR CORONARY ARTERY BLOCAGE AND PATIENT'S QUALITY OF LIFE
}

\author{
(O) Sukovatykh B.S., Nazarenko P.M., Grigorev N.N., Sukovatykh M.B., \\ Gorpinich A.B., Polyanskiy D.B., Mishustin V.N.
}

Kursk State Medical University (KSMU)

3, K. Marx St., Kursk, Kursk region, 305041, Russian Federation

\begin{abstract}
Objective: the aim of the study is to compare the life quality of patients with ischemic heart disease resulted from multivascular coronary artery blockage after CABG surgery on working heart (off-pump coronary artery bypass) and CABG surgery in the environment of artificial circulation and on this basis to determine the most optimal choice of surgery depending on the projected life expectancy of the patient.

Materials and methods. The life quality of 40 patients with ischemic heart disease who had had CABG surgery was analyzed. All patients were separated into two groups, each consisting of 20 patients. Patients in the first group had CABG surgery on the working heart. The second group of patients had surgery on the arrested heart with artificial circulation. 6 and 12 months after surgery, the life quality of patients was assessed using SF-36 questioner.

Results. In comparison with the healthy population of the Russian Federation, patients' physical functioning and mental health before surgery had been decreased to $23.6 \pm 3.3$ and to $10.2 \pm 3.1$ respectively $(\mathrm{p}<0.05)$. 6 months after surgery, the physical functioning of the first group patients increased by $5.7 \pm 1.0$, whereas in the second group by $4.9 \pm 0.7$. Mental health increased by $2.0 \pm 0.4$ and $1.8 \pm 0.4(\mathrm{p}>0.05)$ respectively. 12 months after surgery patients' physical functioning in the first group was higher by $4.9 \pm 0.1$ in the second group and mental health by $3.4 \pm 0.7(p<0.05)$ respectively.

Conclusion. Operations on the working heart should be performed in patients with a short predictable life expectancy with less than $40 \%$ ejection fraction of the left ventricular.

Keywords: ischemic heart disease; multivascular coronary artery blockage; CABG; off-pump coronary artery bypass; working heart; artificial circulation; quality of life.
\end{abstract}

Sukovatykh Boris S. - DM, Professor, Head of Department of General Surgery, KSMU, Kursk. ORCID iD: 0000-0003-2197-8756. E-mail: sukovatykhbs@kursksmu.net (correspondence author)

Nazarenko Petr M. - DM, Professor, Head of Department of Surgical Diseases N 2, KSMU, Kursk. ORCID iD: 0000-0003-4961-1382. E-mail: nazarenkopm@kursksmu.net

Grigorev Nikolay N. - DM, Professor of Department of Surgical Diseases of Post-Graduate Faculty, KSMU, Kursk. ORCID iD: 00000002-4102-1516. E-mail: grigorevnn@kursksmu.net

Sukovatykh Mikhail B. - PhD in Medicine, Associate Professor of Department of General Surgery, KSMU, Kursk. ORCID iD: 0000-0003-1907-4395. E-mail: sukovatykhmb@kursksmu.net

Gorpinich Alexander B. - DM, Professor, Professor of Department of General Surgery, KSMU, Kursk. E-mail: doc.goralex@mail.ru

Polyanskiy Dmitry B. - Resident Physician of Department of General Surgery, KSMU, Kursk. ORCID iD: 0000-0003-3077-8801. E-mail: polyanskydmitriy@yandex.ru

Mishustin Vladimir N. - DM, Professor, Professor of Department of Surgical Diseases of Post-Graduate Faculty, KSMU, Kursk. ORCID iD: 0000-0002-4104-5681. E-mail: vladimirshef2011@yandex.ru

\section{CONFLICT OF INTEREST}

The authors declare the absence of obvious and potential conflicts of interest related to the publication of this article.

\section{SOURCE OF FINANCING}

The authors state that there is no funding for the study.

\section{CONFORMITY WITH THE PRINCIPLES OF ETHICS}

In accordance with the Declaration of Helsinki, voluntary informed consent was obtained from patients to participate in this study; the design was approved by the Regional Ethical
Committee under Kursk state medical university (Protocol No. 5 of 11.05.2015).

\section{AUTHORS CONTRIBUTION}

Sukovatykh B.S. - developing the research concept and design; Nazarenko P.M. - analyzing the investigated literature data; Grigorev N.N. - analyzing the investigated obtained data; Sukovatykh M.B. - writing the manuscript; Gorpinich A.B. analyzing the investigated obtained data; Polyanskiy D.B. - data analysis of SF-36 questionnaire; Mishustin V.N. - analyzing the investigated literature data.

For citation: Sukovatykh B.S., Nazarenko P.M., Grigorev N.N., Sukovatykh M.B., Gorpinich A.B., Polyanskiy D.B., Mishustin V.N. Options of the coronary artery bypass grafting type in patients with multivascular coronary artery blocage and patient's quality of life. Kursk Scientific and Practical Bulletin "Man and His Health". 2019; (3):56-62. DOI: 10.21626/vestnik/2019-3/08. 